\title{
Distribution and cost of wheelchairs and scooters provided by Veterans Health Administration
}

\author{
Sandra L. Hubbard, PhD, OTR/L, ATP; ${ }^{1-2 *}$ Shirley G. Fitzgerald, PhD; ${ }^{3-4}$ Bruce Vogel, PhD; ${ }^{2,5}$ Dean M. \\ Reker, PhD, RN; ${ }^{6-7}$ Rory A. Cooper, PhD; ${ }^{3-4}$ Michael L. Boninger, MD $^{\mathbf{4 , 8}}$ \\ ${ }^{1}$ Occupational Therapy Department, College of Public Health and Health Professions, University of Florida, Gaines- \\ ville, FL; ${ }^{2}$ Rehabilitation Outcomes Research Center of Excellence, North Florida/South Georgia Veterans Health \\ System, Gainesville, FL; ${ }^{3}$ Department of Rehabilitation Science and Technology, School of Health and Rehabilitation \\ Sciences, University of Pittsburgh, Pittsburgh, PA; ${ }^{4}$ Human Engineering Research Laboratories, Department of Veter- \\ ans Affairs (VA) Pittsburgh Healthcare System, Pittsburgh, PA; ${ }^{5}$ Epidemiology and Health Policy Research Depart- \\ ment, College of Medicine, University of Florida, Gainesville, FL; ${ }^{6}$ VA Medical Center, Kansas City, MO; ${ }^{7}$ University \\ of Kansas Medical Center, Kansas City, KS; ${ }^{8}$ Department of Physical Medicine and Rehabilitation, School of Medi- \\ cine, University of Pittsburgh, Pittsburgh, PA
}

\begin{abstract}
During fiscal years 2000 and 2001, the Veterans Health Administration provided veterans with more than 131,000 wheelchairs and scooters at a cost of \$109 million. This national study is the first to investigate Veterans Health Administration costs in providing wheelchairs and scooters and to compare regional prescription patterns. With a retrospective design, we used descriptive methods to analyze fiscal years 2000 and 2001 National Prosthetics Patient Database data (cleaned data set of 113,724 records). Wheelchairs were categorized by function, weight, and adjustability options for meeting individual needs (e.g., axle position, camber, position of wheels, tilt, and recline options). Results displayed a cost distribution that was negatively skewed by low-cost accessories coded as wheelchairs. Of the standard manual wheelchairs, $3.5 \%$ could be considered beyond the customary cost. Regionally, $71 \%$ to $86 \%$ of all wheelchairs provided were manual wheelchairs, $5 \%$ to $11 \%$ were power wheelchairs, and $5 \%$ to $20 \%$ were scooters. The considerable variation found in the types of wheelchairs and scooters provided across Veterans Integrated Service Networks may indicate a need for evidencebased prescription guidelines and clinician training in wheeledmobility technologies.
\end{abstract}

Key words: assistive technology, cost, DME, durable medical equipment, prosthetic, rehabilitation, scooter, vendor, VISN, wheelchair.

\section{INTRODUCTION}

As wheelchair technology becomes increasingly sophisticated and complex, so do decisions regarding who gets what wheelchair. These decisions are significant because manual and power wheelchairs and scooters

\footnotetext{
Abbreviations: ALS = amyotrophic lateral sclerosis, CMS = Centers for Medicare and Medicaid Services, $\mathrm{COPD} / \mathrm{CHF}=$ chronic obstructive pulmonary disorder/chronic heart failure, FY = fiscal year, FY00 = FY 2000, FY01 = FY 2001, HCPCS = Healthcare Common Procedure Coding System, NPPD = National Prosthetics Patient Database, PSAS = Prosthetics and Sensory Aids Service, RESNA = Rehabilitation Engineering and Assistive Technology Society of North America, SCI = spinal cord injury, SD = standard deviation, VA = Department of Veterans Affairs, Veterans Health Administration, VISN = Veterans Integrated Service Network, WC/S = wheelchair and scooter.

* Address all correspondence to Sandra L. Hubbard, PhD, OTR/L, ATP; Occupational Therapy Department, College of Public Health and Health Professions, University of Florida, PO Box 100164, Gainesville, FL 32610; 352-2736125; fax: 352-273-6042. Email: shubbard@phhp.ufl.edu DOI: $10.1682 / J R R D .2006 .10 .0136$
} 
(WC/Ss) were the second, third, and fifth highest Prosthetics and Sensory Aids Service (PSAS) spending totals, respectively, for the first three quarters of fiscal year (FY) 2000. ${ }^{*}$ These WC/S costs translated into a Veterans Health Administration (VHA) cost of more than \$50 million in FY 2000 (FY00).

The VHA, as does any institutional purchaser, seeks to obtain goods at a fair market price commensurate with quality and the discounts normally associated with high purchase volume. However, "assistive devices, like pharmaceuticals, have eluded intensive analysis that adequately addresses fair market pricing” due, according to Render et al., to a lack of assistive technology expertise outside the industry, a lack of expert review of prescription practices, and inconsistencies in billing and reimbursement [1]. "Provision of prosthetic devices needs to be moved from the control of vendors and administrative mavens to the more appropriate control of clinicians and medical needs of the patient" [2]. Evidence to support the provision of prosthetic devices including WC/Ss is inadequate. This study investigates present prescription practices as an initial step toward development of WC/S prescription guidelines.

The first determination a clinician must make when prescribing a WC/S is whether to provide a manual or power device. Clinical practice guidelines for the prescription of motorized WC/Ss are available to Department of Veterans Affairs (VA) clinicians. While these guidelines will aid the decision-making process regarding provision of manual versus power devices, the clinician must still decide which type of device, manual or power wheelchair, is most appropriate. Because of rapid technological advances, many clinicians are making decisions about technology that did not exist when they received their training. Staying abreast of advances in wheeled mobility technology is challenging even for clinicians with seating and mobility expertise. Concurrently, demand for WC/Ss is increasing because of Operation Enduring Freedom and Operation Iraqi Freedom injuries, an aging veteran population, and the increased visibility of wheelchair users because of the decreased stigma resulting from the Americans with Disabilities Act and the Disability Rights Movement. Clinician training can affect variation in the provision of WC/Ss to veterans.

*Fred Downs, Chief Consultant, Prosthetics and Sensory Aids Service, written communication, November 2000.
Variation not based on veteran need should be identified to increase equitable provision of WC/Ss to veterans.

Our first objective was to describe regional patterns of WC/S provision, e.g., whether more power wheelchairs are provided in some areas and more manual wheelchairs in others. Our second objective was to describe the range in cost of specific WC/Ss. We hypothesize that if we can enhance prescription guidelines, then prescription practice will be more consistent from site to site and WC/S expenditures will be more homogeneous. The anticipated result of consistency and homogeneity is that more veterans will be provided WC/Ss and, thus, provision will be more equitable. Further, if patient needs and WC/S capabilities are better matched, a higher quality of care and greater efficiency can be achieved. Information from this study could guide VHA policy makers' decisions and direct follow-up research studies designed to more thoroughly investigate the nature of the observed variations.

\section{METHODS}

\section{Design and Population}

This study, approved as exempt by the VA Pittsburgh Healthcare System and the University of Pittsburgh Institutional Review Boards, relied on existing administrative data collected by the VHA and the PSAS on patients receiving care in the VA system. Using a retrospective design, we extracted records from the National Prosthetics Patient Database (NPPD) of veterans who received wheelchairs or scooters from the VHA during FY00 or FY 2001 (FY01).

\section{Data Management}

The VHA PSAS developed the NPPD to track WC/Ss and other prosthetic expenditures. In addition, the NPPD allows review of prescription practices among clinical teams, facilities, and Veterans Integrated Service Networks (VISNs). Housed at the VA Information Resource Center in Hines, Illinois [3], the NPPD contains information on every device issued to veterans, including a description of the device, prescribing facility and VISN, type of service (initial issue, replacement, spare, or repair), date, cost, and vendor. Data cleaning was performed according to decision rules developed during a collaborative validity study of the NPPD [4]. We further cleaned cost data by excluding 3,799 observations with missing cost values and 5,639 observations with $\$ 0$ cost 
values. Following these exclusions, 121,774 observations remained for FY00 and FY01 combined. Healthcare Common Procedure Coding System (HCPCS) codes (see following section for definition) with five or less observations over the 2-year study period were excluded, leaving 64 HCPCS codes and 121,762 observations. HCPCS code E1065 $(n=161)$ observations were also deleted because of inconsistent coding; i.e., HCPCS code E1065 is intended to described a "power attachment," but when we considered the description, vendor, and cost fields, the code appeared to be used more as a miscellaneous category for power devices.

Wheelchair costs that were too low to represent wheelchairs or scooters were assumed to be either accessories or coding errors and were excluded as invalid observations, leaving 113,724 records. We determined low values for exclusion based on HCPCS code, description of the device, market value, and the VHA contract amount, if known. For example, observations with HCPCS code K0001 (standard manual wheelchair) with a cost of less than $\$ 100$ were excluded since the VA competitive bid contract cost for K0001 wheelchairs is greater than \$100. Similarly, observations with HCPCS codes K0010-K0014 (power wheelchairs) with a cost of less than $\$ 1,000$ were excluded since, based on market value, these observations were more likely to be accessories than wheelchairs.

\section{Operational Definitions}

Healthcare Common Procedure Coding System Codes

WC/Ss sold in the United States must be approved by the Food and Drug Administration [5]. The Food and Drug Administration forwards information on the wheelchair application to the Centers for Medicare and Medicaid Services (CMS). CMS assigns the wheelchair an HCPCS code, a standard code developed for reimbursement purposes [6]. HCPCS codes are used to identify devices in the NPPD.

\section{Wheelchair Type}

Because of the large quantity of unique wheelchairrelated HCPCS codes in the NPPD $(n=71)$, analysis of each wheelchair code was not feasible; therefore, for this study, we assigned the HCPCS codes used to describe wheelchairs in the NPPD to one of eight types based on function, weight, and adjustability (i.e., M1, M2, M3, M4, P1, P2, P3, and S1). Adjustability (axle position, camber for manual wheelchairs, position of wheels, tilt, recline options, etc.) is essential for customizing a wheelchair to individual needs. Table 1 lists the assigned wheelchair

Table 1.

Operational definitions of dependent variable: Wheelchair (WC) or scooter type.

\begin{tabular}{|c|c|c|}
\hline $\begin{array}{c}\text { HCPCS Code, Description } \\
\end{array}$ & WC/Scooter Type* & $\begin{array}{r}\text { Description } \\
\end{array}$ \\
\hline K0001, Manual WC Depot & M1 & >36 lb, nonadjustable, depot WC \\
\hline K0002, Manual WC Hemiplegia & & >36 lb; nonadjustable, lower seat only; depot WC \\
\hline K0003, Manual WC Lightweight & & 36 lb, nonadjustable, lightweight, depot WC \\
\hline K0004, Manual WC Lightweight & M2 & $\begin{array}{l}<34 \text { lb; adjustable seat/back height, some adjustment in axle; } \\
\text { high strength; lightweight; rehabilitation WC }\end{array}$ \\
\hline K0005, Manual WC Ultralightweight & M3 & $\begin{array}{l}<30 \mathrm{lb} \text {; adjustable seat/back height/axle/camber; ultralight- } \\
\text { weight WC }\end{array}$ \\
\hline $\begin{array}{l}\text { K0006-7, Manual WC Heavy Duty } \\
\text { K0009, Manual WC Other }\end{array}$ & M4 & Miscellaneous manual WC \\
\hline K0010, Power WC & $\mathrm{P} 1$ & $\begin{array}{l}\text { Nonadjustable, seat height only; standard weight; nonpro- } \\
\text { grammable controls }\end{array}$ \\
\hline $\begin{array}{l}\text { K0011, Power WC } \\
\text { K0012, Power WC }\end{array}$ & $\mathrm{P} 2$ & Miscellaneous power WC \\
\hline K0014, Power WC & P3 & Custom power WC, other motorized WC base \\
\hline E1230, Scooter & S1 & Scooter \\
\hline
\end{tabular}


types. The NPPD includes 71 HCPCS codes (E- and Kcodes) for WC/Ss. For simplicity, only the wheelchair Kcodes, which represent 84 percent of all wheelchairs prescribed, are included in Table 1. A complete listing of E- and K-codes can be found in the Appendix (available online only at http://www.rehab.research.va.gov/). The Ecode for scooters is included in Table 1 because no Kcode exists for scooters.

\section{Cost}

We determined cost, the first unit of analysis, as follows. A wheelchair accessory was added to the cost of the wheelchair if the date the accessory was entered into the system ("createdate") was within \pm 10 days of the wheelchair "createdate." "Createdate" is the date the device was ordered and logged into the Veterans Health Information Systems and Technology Architecture Prosthetics Package [3] and is uploaded into the NPPD. Wheelchair accessories in the NPPD can refer to removable arm/leg rests, seating systems, etc.

\section{Veterans Integrated Service Network}

The second unit of analysis was geographic region, or VISN. In FY00 and FY01, the approximately 1,127 VHA facilities were organized into 22 VISNs [7]. A VISN may include more than one state (e.g., in New England), and large states (e.g., Texas) may have more than one VISN. In this study, we used VISNs as the geographical unit of comparison since they are the geographical unit used in all VHA databases, including the NPPD.

We performed frequency counts for categorical variables and calculated the mean and standard deviation (SD) for continuous variables to describe the population (all veterans who received a wheelchair or scooter from the VHA during FY00-FY01). The following diagnoses were included: amyotrophic lateral sclerosis (ALS), multiple sclerosis, spinal cord injury (SCI)-tetraplegia, SCIparaplegia, stroke, traumatic brain injury, Parkinson's disease, amputation, chronic obstructive pulmonary disorder/chronic heart failure (COPD/CHF), and arthritis. An "other" category was established for primary diagnoses other than those listed. These diagnoses were selected based on frequency as a primary diagnosis and likelihood of being the diagnosis most relevant to the wheelchair or scooter prescription [8]. We used frequency counts to describe the number and proportion of WC/Ss provided each FY.

We calculated mean, median, SD, minimum, and maximum values to describe the number and cost of WC/Ss provided per HCPCS code. Finally, we used frequency counts to describe the numbers and proportions of manual wheelchairs, power wheelchairs, and scooters provided per VISN. We then created a ratio of number of devices provided per VISN to number of veterans served per VISN to compare mean number of devices provided per veterans served across VISNs. SAS ${ }^{\circledR}$ (SAS Institute Inc, Cary, North Carolina) software was used for all analyses.

\section{RESULTS}

Table 2 displays the demographic characteristics of the study population of veterans receiving $\mathrm{WC} / \mathrm{Ss}$. The veteran in this population was most typically a white male, approximately 68 years old with a primary diagnosis of COPD/CHF. The racial/ethnic distribution was 6 percent Hispanic, 14 percent African American, and 65 percent

Table 2.

Demographic characteristics by fiscal year (FY). All data shown as percentages unless otherwise indicated.

\begin{tabular}{lcc}
\hline \multicolumn{1}{c}{ Variable } & FY 2000 & FY 2001 \\
\hline Unique Veterans (No.) & 49,026 & 48,519 \\
Sex (Male) & 94 & 94 \\
Race/Ethnicity & 6 & \\
Hispanic & $<1$ & 61 \\
American Indian & 14 & 13 \\
African American & $<1$ & $<1$ \\
Asian & 65 & 62 \\
White & 15 & 18 \\
Unknown & & \\
Diagnosis & $<1$ & $<1$ \\
Amyotrophic Lateral Sclerosis & 3 & 3 \\
Multiple Sclerosis & 7 & 6 \\
Spinal Cord Injury (tetraplegia or & & \\
$\quad$ paraplegia) & 16 & 14 \\
Stroke & $<1$ & $<1$ \\
Traumatic Brain Injury & 3 & 3 \\
Parkinson's Disease & 4 & 3 \\
Amputation & 23 & 23 \\
Chronic Obstructive Pulmonary & & \\
$\quad$ Disorder/Chronic Heart Failure & & 12 \\
Arthritis & 31 & $68.2 \pm 12.1$ \\
Other & $67.4 \pm 13.1$ & 1 \\
Age (mean \pm SD) & 2 & \\
Demographic Data Unavailable & & \\
\hline SD = standard deviation. & & \\
\hline \hline
\end{tabular}


white. Nearly one-quarter (23\%) of the veterans in this study had a primary diagnosis of COPD/CHF; approximately 15 percent were diagnosed with stroke, 11 percent with arthritis, and 7 percent with SCI (SCI-paraplegia and SCI-tetraplegia combined). The mean \pm SD age in years increased from $67.4 \pm 13.1$ in FY00 to $68.2 \pm 12.1$ in FY01.

Table 3 displays the number and corresponding percentage of WC/Ss provided by the VHA in FY00 and FY01. The proportion of manual wheelchairs provided remained relatively stable from FY00 to FY01 (79\% vs $77 \%$, respectively). The proportion of power wheelchairs provided increased from 8 percent of all devices in FY00 to 14 percent in FY01; the proportion of scooters decreased from 13 percent in FY00 to 9 percent in FY01.

Table 4 displays the number and cost (mean, median, $\mathrm{SD}$, minimum, and maximum) of the most frequently provided WC/Ss. For brevity, only the values for WC/Ss

Table 3.

Wheelchairs and scooters provided by Veterans Health Administration.

\begin{tabular}{|c|c|c|c|c|c|c|c|}
\hline \multirow[b]{2}{*}{ Fiscal Year } & \multirow{2}{*}{$\begin{array}{c}\text { Total Devices } \\
\text { (No.) }\end{array}$} & \multicolumn{4}{|c|}{ Wheelchairs } & \multirow{2}{*}{\multicolumn{2}{|c|}{ Scooters }} \\
\hline & & \multicolumn{2}{|c|}{ Manual } & \multicolumn{2}{|c|}{ Power } & & \\
\hline 2000 & 63,351 & 49,898 & 79 & 5,309 & 8 & 8,144 & 13 \\
\hline
\end{tabular}

Table 4.

Number supplied and cost of wheeled mobility devices per Healthcare Common Procedure Coding System (HCPCS) code for fiscal years 2000 and 2001 combined. Note that HCPCS code descriptions are shown exactly as provided by Centers for Medicare and Medicaid Services.

\begin{tabular}{|c|c|c|c|c|c|c|c|}
\hline \multirow{2}{*}{$\begin{array}{c}\text { Type/HCPCS } \\
\text { Code }\end{array}$} & \multirow{2}{*}{$\begin{array}{c}\text { HCPCS } \\
\text { Code Description }\end{array}$} & \multirow{2}{*}{$\begin{array}{l}\text { Devices } \\
\text { (No.) }\end{array}$} & \multicolumn{5}{|c|}{ National Cost (\$) } \\
\hline & & & Mean & Median & SD & Min & Max \\
\hline \multicolumn{8}{|c|}{ M1 Standard Manual Wheelchair } \\
\hline E1140 & W/c standard detach a & 2,441 & 214 & 170 & 209 & 100 & 7,057 \\
\hline E1150 & W/c standard w/leg r & 1,384 & 291 & 211 & 346 & 100 & 9,186 \\
\hline E1260 & W/c lightwt foot rest & 1,219 & 323 & 244 & 250 & 107 & 3,090 \\
\hline K0001 & Standard wheelchair & 43,429 & 204 & 163 & 206 & 100 & 13,667 \\
\hline \multicolumn{8}{|c|}{ M2 Lightweight Rehabilitation Manual Wheelchair } \\
\hline E1088 & W/c lightweight det a & 1,068 & 700 & 455 & 748 & 200 & 10,565 \\
\hline K0003 & Lightweight wheelchair & 8,773 & 495 & 356 & 437 & 100 & 9,045 \\
\hline K0004 & High strength ltwt whlchr & 15,318 & 480 & 362 & 381 & 200 & 12,346 \\
\hline \multicolumn{8}{|c|}{ M3 Ultralightweight Manual Wheelchair } \\
\hline K0005 & Ultralightweight wheelchair & 3,076 & 1,205 & 1,067 & 649 & 400 & 11,514 \\
\hline \multicolumn{8}{|c|}{ M4 Miscellaneous Manual Wheelchair } \\
\hline K0006 & Heavy duty wheelchair & 2,331 & 626 & 450 & 585 & 108 & 9,843 \\
\hline K0007 & Extra heavy duty wheelchair & 1,322 & 727 & 496 & 600 & 116 & 9,595 \\
\hline K0008 & Custom manual wheelchair/base & 1,068 & 1,070 & 917 & 951 & 201 & 10,422 \\
\hline \multicolumn{8}{|c|}{ P1 Standard Power Wheelchair } \\
\hline E1211 & W/c motorized w/det & 1,268 & 4,548 & 3,804 & 2,608 & 1,056 & 19,412 \\
\hline E1212 & W/c motorized w full & 1,127 & 4,576 & 4,200 & 2,469 & 1,031 & 17,728 \\
\hline \multicolumn{8}{|c|}{ P2 Miscellaneous Power Wheelchair } \\
\hline K0011 & Std wt pwr whlchr w control & 2,743 & 4,323 & 3,897 & 2,238 & 1,000 & 25,852 \\
\hline \multicolumn{8}{|c|}{ P3 Custom Power Wheelchair } \\
\hline K0014 & Other power whlchr base & 1,759 & 5,237 & 4,504 & 2,947 & 1,000 & 24,459 \\
\hline \multicolumn{8}{|l|}{ S1 Scooter } \\
\hline E1230 & Powered operated vehicle & 14,721 & 2,047 & 1,935 & 803 & 900 & 17,883 \\
\hline
\end{tabular}


most frequently provided nationally $(n>1,000)$ are reported. Note that we present the HCPCS code descriptions exactly as they are provided by CMS. The Appen. dix provides the number and cost data on all WC/Ss provided. The most frequently provided WC/S was the K0001 standard manual wheelchair $(n=43,429)$, followed by the K0004 lightweight rehabilitation wheelchair $(n=15,318)$, and then the E1230 scooter $(n=$ 14,721).

The median cost of the K0001 standard manual wheelchair was $\$ 163$, with minimum and maximum values of $\$ 100$ and $\$ 13,667$, respectively. We present median rather than mean costs because the mean values were skewed by excessively high costs, most likely representing coding error, e.g., custom power wheelchairs coded as standard manual wheelchairs. Approximately 10 percent $(n=4,264)$ of the standard wheelchairs cost more than $\$ 300$ and 1 percent $(n=400)$ cost more than $\$ 1,000$. All VISNs had supplied standard manual wheelchairs that cost more than $\$ 1,000$. The range of standard manual wheelchairs exceeding $\$ 1,000$ across VISNs was 1 to 14 percent.

The median cost of the K0004 lightweight rehabilitation wheelchair was $\$ 362$, with minimum and maximum values of $\$ 200$ and $\$ 12,346$, respectively. Approximately 7 percent $(n=1,132)$ of the lightweight rehabilitation wheelchairs cost more than $\$ 1,000$, with less than 1 percent $(n=10)$ costing more than $\$ 5,000$. The range of lightweight rehabilitation manual wheelchairs exceeding $\$ 1,000$ across VISNs was 1 to 12 percent.

The median cost of the E1230 scooter was $\$ 1,935$, with minimum and maximum values of $\$ 900$ and $\$ 17,883$, respectively. Less than 1 percent $(n=129)$ of the scooters cost more than $\$ 5,000$ and only 21 scooters cost more than $\$ 10,000$. All but two VISNs had scooter costs exceeding $\$ 5,000$. The range of scooters exceeding $\$ 1,000$ across VISNs was 0 to 19 percent.

The K0004 lightweight rehabilitation wheelchair had the highest proportion of outlier costs: 8 percent of the K0004 wheelchairs purchased cost twice the national mean or more. In comparison, only 2 percent of K0001 wheelchairs and 2 percent of scooters cost twice the national mean or more.

Tables 5 and 6 display the percentages of WC/Ss provided across VISNs for FY00 and FY01 combined: Table 5 displays the distribution of manual wheelchairs, power wheelchairs, and scooters, whereas Table 6 displays the distribution of all eight WC/S types. The Figure provides a map of all VISNs. The range in percentage of WC/Ss provided across VISNs was 71 to 86 percent manual wheelchairs, 5 to 11 percent power wheelchairs, and 5 to 20 percent scooters (Table 5). Thus for all VISNs, at least 71 percent of the wheelchairs provided were manual. Seven VISNs provided the same or nearly the same proportion of power wheelchairs as scooters (e.g., $10 \%$ vs $10 \%$, respectively; $8 \%$ vs $7 \%$, respectively). Three VISNs provided a higher proportion of power wheelchairs than scooters (i.e., $9 \%$ vs $5 \%$, respectively; $11 \%$ vs $8 \%$, respectively; and $10 \%$ vs $7 \%$, respectively). Eleven VISNs provided a higher proportion of scooters than power wheelchairs (e.g., $11 \%$ vs $8 \%$, respectively; $17 \%$ vs $10 \%$, respectively) (Table 5). The VISNs that provided higher proportions of manual wheelchairs $(82 \%-86 \%)$ tended to provide either more power wheelchairs or approximately the same proportion of power wheelchairs as scooters. Conversely, VISNs that provided a lesser proportion of manual wheelchairs $(71 \%-81 \%)$ provided a higher proportion of scooters than power wheelchairs. This finding suggests that the VISNs that provide more power mobility devices provide more scooters than power wheelchairs.

Column 7 of Table 5 displays the total number of WC/Ss provided in FY00 and FY01 across VISNs. Because reporting the number of devices provided per VISN does not consider the number of veterans served per VISN, the number of veterans served per VISN is included in the table (column 9). The ratio of veterans served to devices provided was then calculated (column 10), resulting in a range from 38:1 to $95: 1$ and a mean of 63:1. Unfortunately, FY00 enrollment data by VISN could not be located, so the ratio of number of veterans served to number of devices provided is for FY01 only.

Table 6 displays the geographical variation in WC/S provision in more detail, which enables geographical comparison of the provision of high-end versus low-end manual and power wheelchairs.

\section{Manual Wheelchairs}

As few as 20 percent and as many as 66 percent of all WC/Ss provided by individual VISNs were low-end standard manual wheelchairs (M1: K0001-K0003); the range of provision of high-end manual wheelchairs (M3: K0005) was 1 to 9 percent. VISNs that provided more lightweight rehabilitation wheelchairs tended to provide fewer standard manual wheelchairs and fewer ultralightweight manual wheelchairs. 
Table 5.

Distribution of all types of wheeled mobility devices across Veterans Integrated Service Network (VISN).

\begin{tabular}{|c|c|c|c|c|c|c|c|c|c|}
\hline \multirow[b]{2}{*}{ VISN } & \multicolumn{6}{|c|}{ FY00 and FY01 Combined } & \multicolumn{3}{|c|}{ FY01 Only* } \\
\hline & $\begin{array}{c}\% \% \\
\text { Manual }\end{array}$ & $\begin{array}{c}\% \\
\text { Power }\end{array}$ & $\begin{array}{c}\% \\
\text { Scooter }\end{array}$ & $\begin{array}{c}\text { \% Power \& } \\
\text { Scooter }\end{array}$ & $\begin{array}{l}\text { Power: } \\
\text { Manual }\end{array}$ & $\begin{array}{c}\text { No. } \\
\text { Devices }\end{array}$ & $\begin{array}{c}\text { No. } \\
\text { Devices }\end{array}$ & $\begin{array}{l}\text { No. } \\
\text { Users }\end{array}$ & $\begin{array}{c}\text { No. Veterans: } \\
\text { Devices Provided }^{\dagger}\end{array}$ \\
\hline 1 & 81 & 8 & 11 & 19 & 0.23 & 3,100 & 2,138 & 199,624 & 93 \\
\hline 2 & 80 & 5 & 15 & 20 & 0.25 & 2,456 & 1,493 & 128,031 & 86 \\
\hline 3 & 86 & 9 & 5 & 14 & 0.16 & 3,595 & 1,877 & 199,108 & 106 \\
\hline 4 & 73 & 10 & 17 & 27 & 0.37 & 4,596 & 2,706 & 245,980 & 91 \\
\hline 6 & 74 & 11 & 15 & 26 & 0.35 & 6,211 & 3,622 & 203,590 & 56 \\
\hline 7 & 81 & 11 & 8 & 19 & 0.23 & 9,074 & 4,932 & 245,641 & 50 \\
\hline 8 & 81 & 7 & 12 & 19 & 0.23 & 13,852 & 8,282 & 410,808 & 50 \\
\hline 9 & 80 & 10 & 10 & 20 & 0.25 & 6,169 & 3,659 & 208,003 & 57 \\
\hline 10 & 75 & 8 & 17 & 25 & 0.33 & 2,514 & 1,523 & 141,955 & 93 \\
\hline 15 & 86 & 6 & 8 & 14 & 0.16 & 4,343 & 2,577 & 180,885 & 70 \\
\hline 16 & 71 & 9 & 20 & 29 & 0.41 & 15,203 & 8,787 & 361,625 & 41 \\
\hline 17 & 76 & 8 & 16 & 24 & 0.32 & 7,100 & 4,164 & 191,189 & 46 \\
\hline 18 & 85 & 7 & 8 & 15 & 0.18 & 4,305 & 2,518 & 207,488 & 82 \\
\hline 19 & 83 & 10 & 7 & 17 & 0.20 & 2,866 & 1,708 & 118,361 & 69 \\
\hline 20 & 82 & 9 & 9 & 18 & 0.22 & 4,363 & 2,647 & 173,004 & 65 \\
\hline 21 & 73 & 8 & 19 & 27 & 0.37 & 3,714 & 2,371 & 191,801 & 81 \\
\hline 22 & 80 & 10 & 10 & 20 & 0.25 & 5,925 & 3,463 & 217,135 & 63 \\
\hline
\end{tabular}

*FY2000 Veterans Health Administration user data not available.

${ }^{\dagger}$ Mean \pm standard deviation $=63 \pm 16$.

FY = fiscal year, FY00 = FY 2000, FY01 = FY 2001.

\section{Power Wheelchairs}

Less geographic variation was found in the provision of power wheelchairs. Three VISNs provided the same proportion of low-end as high-end power wheelchairs, seven provided a larger proportion of high-end power wheelchairs, and the remaining twelve VISNs provided a larger proportion of low-end power wheelchairs.

\section{Manual Wheelchairs Versus Power Wheelchairs and Scooters}

The VISN that provided the highest percentage of low-end manual wheelchairs also provided the highest percentage of high-end power wheelchairs. We noted a trend in which the four VISNs providing the highest proportion of low-end manual wheelchairs provided a relatively higher proportion of power wheelchairs and relatively lower proportion of scooters $(>5 \%$ standard power wheelchairs and $<14 \%$ scooters). Another trend observed was that the four VISNs providing a lower proportion of low-end manual wheelchairs provided a relatively higher proportion of scooters ( $>15 \%$ scooters). Two of these four VISNs also provided a relatively higher proportion of standard power wheelchairs $(6 \%$ and $7 \%$ ). The three VISNs providing the highest proportion of custom power wheelchairs $(>4 \%)$ also provided relatively high proportions of ultralightweight manual wheelchairs (>3\%).

\section{DISCUSSION}

This national study describes variation in type of $\mathrm{WC} /$ Ss provided to veterans across geographic regions, or VISNs, and variation in cost of similar WC/Ss. Most studies of wheelchair use consider wheelchairs as one category, i.e., a participant either does or does not use a 
JRRD, Volume 44, Number 4, 2007

Table 6.

Distribution (\%) of all types of wheeled mobility devices by Veterans Integrated Service Network (VISN).

\begin{tabular}{|c|c|c|c|c|c|c|c|c|c|}
\hline \multirow{2}{*}{ VISN } & \multicolumn{4}{|c|}{ Manual } & \multicolumn{5}{|c|}{ Power } \\
\hline & M1 & M2 & M3 & M4 & P1 & P2A & P2B & P3 & S1 \\
\hline 1 & 20 & 54 & 3 & 4 & 1 & 4 & 1 & 2 & 11 \\
\hline 2 & 57 & 11 & 5 & 7 & 4 & $<1$ & $<1$ & $<1$ & 15 \\
\hline 3 & 62 & 15 & 3 & 6 & 5 & 2 & $<1$ & 2 & 5 \\
\hline 4 & 41 & 22 & 1 & 9 & 7 & 2 & $<1$ & 1 & 17 \\
\hline 5 & 54 & 13 & 3 & 6 & 2 & 2 & $<1$ & 2 & 18 \\
\hline 6 & 38 & 24 & 1 & 10 & 3 & 6 & $<1$ & 2 & 15 \\
\hline 7 & 53 & 17 & 3 & 8 & 1 & 5 & 1 & 4 & 8 \\
\hline 8 & 55 & 19 & 2 & 5 & 5 & 2 & $<1$ & $<1$ & 12 \\
\hline 9 & 66 & 3 & 4 & 7 & $<1$ & 1 & 3 & 6 & 10 \\
\hline 10 & 27 & 42 & $<1$ & 5 & 6 & 1 & $<1$ & 1 & 17 \\
\hline 11 & 63 & 6 & 1 & 11 & 3 & 1 & $<1$ & 1 & 14 \\
\hline 12 & 47 & 27 & 5 & 6 & 1 & 2 & 1 & 4 & 8 \\
\hline 13 & 51 & 23 & 1 & 7 & 3 & 1 & 3 & 2 & 10 \\
\hline 14 & 50 & 10 & 5 & 10 & 2 & 2 & $<1$ & 3 & 17 \\
\hline 15 & 51 & 25 & 1 & 9 & 4 & 1 & $<1$ & $<1$ & 8 \\
\hline 16 & 60 & 6 & 1 & 4 & 5 & 2 & $<1$ & 1 & 20 \\
\hline 17 & 58 & 6 & 4 & 7 & 3 & 2 & 1 & 3 & 16 \\
\hline 18 & 55 & 23 & 2 & 5 & 3 & 1 & $<1$ & 2 & 8 \\
\hline 19 & 50 & 23 & 2 & 7 & 2 & 6 & $<1$ & 2 & 7 \\
\hline 20 & 57 & 16 & 1 & 8 & 5 & 3 & $<1$ & 2 & 9 \\
\hline 21 & 53 & 13 & 4 & 3 & 2 & 2 & $<1$ & 3 & 19 \\
\hline 22 & 49 & 17 & 9 & 5 & 2 & 4 & 1 & 3 & 11 \\
\hline
\end{tabular}

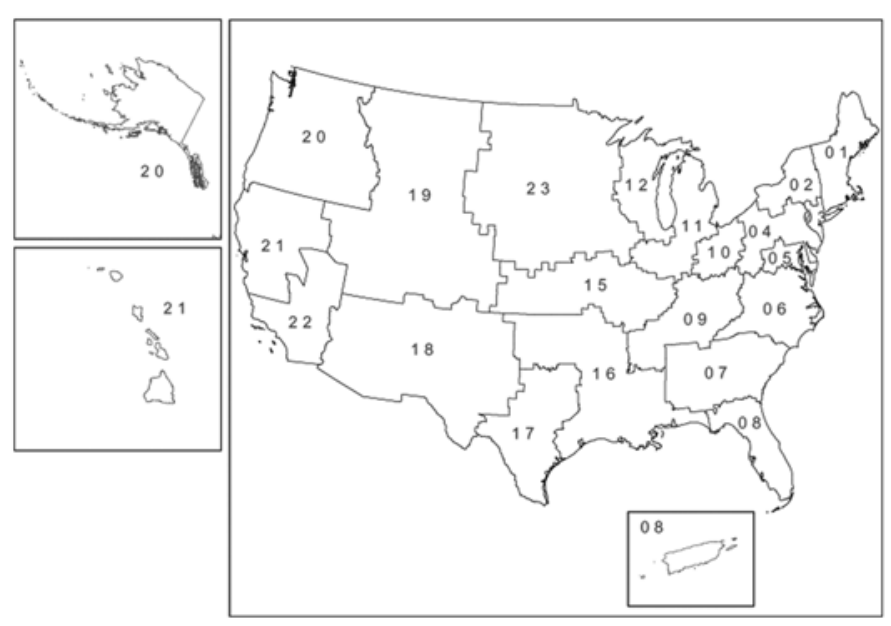

Figure.

Veterans Integrated Service Networks. Map courtesy of Dr. Diane C. Cowper Ripley and Mr. Eric R. Litt, Rehabilitation Outcomes Research Center of Excellence, North Florida/South Georgia Veterans Health System, Gainesville, Florida. wheeled mobility device [9-11]. The problem with these studies is that they assume all wheelchairs are created equal when, in fact, quite a range in cost, adjustability, and features exists. Studies that have compared types of wheelchairs have focused on durability [12-15] rather than on provision. Only one other study was found that examined the provision of wheelchairs by type [16]. That population included 412 individuals with SCI who were receiving services from the SCI Model Systems. Of the manual wheelchairs users, only 3 percent used a standard manual wheelchair (K0001-K0004); 97 percent used a customizable lightweight wheelchair (K0005). Of the power wheelchair users, 46 percent used a standard chair with programmable controls (K0011) and 54 percent used customized power wheelchairs (K0014). No participants used a standard power wheelchair (K0010) [16]. This study, however, did not consider cost or geographic variation and was limited to individuals with SCI. 


\section{Cost}

The most frequently provided type of wheelchair was the standard manual wheelchair $(n=54,358,48 \%)$. The majority, or 80 percent $(n=43,429)$, of wheelchairs included in the "standard manual category" were coded K0001. See the Appendix for a complete listing. The mean cost for the K0001 was \$204 compared with a median cost of $\$ 163$. The VA competitive bid price for the K0001 in FY01 was approximately \$114, unaccessorized/base cost. ${ }^{*}$ Of the K0001 observations in the cleaned cost data set, approximately 75 percent cost \$100-\$199, 15 percent \$200-\$299, 2.5 percent $>\$ 500$, and 1 percent $>\$ 950(n=471)$. Costs in the $\$ 500$ range can be justified by additional accessories such as tilt, recline, delivery, and setup charges.

During FY01, 8 and 13 percent of the WC/Ss provided by the VHA were power WC/Ss, respectively. The mean cost for scooters was $\$ 2,047$ and the median cost was $\$ 1,935$. These costs represent a customary range for scooters. Typically, scooters cannot be customized, e.g., by adding removable arm/leg rests, custom seating. The range for custom power wheelchairs, $\$ 1,000-\$ 24,459$, was also reasonable. Costs less than $\$ 2,000$ were likely to represent accessories, such as tilt or a seating system. While the mean cost for a custom power wheelchair was $\$ 5,237$, a cost of $\$ 24,459$ is perhaps a customary price, e.g., to construct a chair for a veteran with ALS. Nationally, only four power wheelchairs exceeded $\$ 20,000$ over a 2-year period; 93 percent of power wheelchairs cost less than $\$ 10,000(n=1,631)$.

The differences between the mean and median costs, with median values less than mean values, indicate positive skew possibly due to variation in coding and data entry. For example, wheelchair accessories (e.g., detachable leg rest, seating system, battery/battery charger, delivery, and setup costs) were coded several ways: (1) as the wheelchair they accompanied rather than as accessories, (2) as part of the total price for the wheelchair, or (3) as accessories, with the wheelchair base price coded as a wheelchair.

Setup and delivery charges could potentially be included in the price of the device, or itemized. Setup is a particularly important safety factor in the provision of powered devices with programmable controls to veter-

\footnotetext{
*Fred Downs, Chief Consultant, Prosthetics and Sensory Aids Service, written communication, 2002.
}

ans. Programmable controls allow the responsiveness and speed of the wheelchair to be adjusted to the individual veteran's needs. Factory settings may be inappropriate. Errors in factory settings can occur and can cause, for example, a chair to drive at excessive speed with only a light touch to the joystick. Manual wheelchairs also require setup. Axle and camber positions and seat and leg/foot rest angles must be adjusted for each user (for wheelchairs that allow these adjustments) to maximize propulsion efficiency and minimize secondary injuries to the shoulder, elbow, and wrist joints [17-20].

Data entry varied with method of purchase. For example, WC/Ss could be provided from stock, customized by a VA-employed technician, customized by a vendor, or purchased via the General Services Administration Web site. Future studies could be designed to identify and rank the most effective purchase options for WC/Ss.

Another factor contributing to the skewed cost distribution was the VHA reissue policy that states "whenever possible, reclaimed wheelchairs that have been restored will be reissued" (1173.6) [21]. This policy has potentially tremendous cost- and time-saving implications and benefits for veterans. Typically, reissued wheelchairs were assigned a cost of $\$ 0$ - observations that were removed during data cleaning. In FY01, a policy change designated that reissued chairs be assigned a cost equal to one-half the original price.

A lapse of 6 to 16 weeks is not unusual between order and delivery of a custom power or manual wheelchair. During this time, the intended user's motor skills may change. A veteran with ALS, for example, may not be able to use a wheelchair that had been prescribed 4 weeks prior. Providing this veteran with an on-hand (reissued) wheelchair could improve his or her quality of care versus having no wheelchair or an obsolete wheelchair while waiting for the new one. In addition, one VHA wheelchair purchase can benefit more than one veteran. Frequently, expensive wheelchairs can no longer be used by veterans because of changing needs and abilities. When the wheelchairs remain in good condition, recycling these devices becomes very cost effective. In addition, devices are sometimes prescribed for veterans who do not have progressive conditions but, nonetheless, once the devices arrive, they do not meet their needs. In a qualitative study of individuals with SCI, Kittel et al. found that participants receiving their first wheelchair (as inpatients) had little insight into their own needs and expectations upon reentering community life, plus they lacked the ability to 
learn from other wheelchair users. Their second wheelchair was more satisfactory than the first because they had had an opportunity to realize their wheelchair needs and lifestyle preferences [15]. An ill-prescribed wheelchair for one veteran could be the perfect wheelchair for another.

\section{Provision by Type}

The results of this study identified and quantified national variation in the provision of manual and power WC/Ss. Across VISNs, the percentage of manual wheelchairs provided during FY00 and FY01 ranged from 71 to 80 percent; inversely, the percentage of power devices (power WC/Ss) provided ranged from 20 to 29 percent. Typically, the variation in power devices provided was driven by the percentage of scooters provided; i.e., for 19 out of 22 VISNs, providing a relatively higher percentage (20\%-29\%) of power devices was associated with providing a relatively higher percentage $(11 \%-20 \%)$ of scooters. Inversely, providing a relatively lower percentage $(15 \%-19 \%)$ of power devices was associated with providing a relatively lower percentage $(5 \%-10 \%)$ of scooters. The shift in provision of power devices described in Table 3 is interesting. During FY00, 8 percent of the devices provided were power wheelchairs and 13 percent were scooters. In FY01, this pattern shifted to 14 percent power wheelchairs and 9 percent scooters. A current study is underway to determine whether these same patterns are present in the WC/Ss provided to veterans by Medicare.

The variation in the percentage of manual (71\%$80 \%)$ and power $(20 \%-29 \%)$ devices provided across VISNs became far more extreme when types of manual and power devices were considered. When we looked at a data subset of manual wheelchairs only, the provision of standard manual wheelchairs ranged from 24 (VISN 1) to 83 percent (VISN 9) (data not presented in tables), the provision of lightweight manual wheelchairs ranged from 4 (VISN 9) to 67 percent (VISN 1), and the provision of ultralightweight manual wheelchairs ranged from $\leq 1$ percent (five VISNs) to 11 percent (VISN 22). Similarly, when we looked at a data subset of power devices only (data not presented in tables), the provision of standard power wheelchairs ranged from 2 (VISN 9) to 33 percent (VISN 3), the provision of custom power wheelchairs ranged from 2 (VISN 2) to 28 percent (VISN 9), and the provision of scooters ranged from 33 (VISN 3) to 77 percent (VISN 2).
One should note that this study examined FY00FY01 data. At that time, little guidance was available to VA clinicians as to what qualified a patient for a given prosthetic device. Since then, the VA has made available clinical practice guidelines on the provision of power mobility devices, including guidelines for the provision of a high-technology power wheelchair (the iBot, http:// www.ibotnow.com/ibot/index.html). While guidelines are available on provision of manual versus power devices and provision of types of power devices, no known guidelines exist on provision of manual or power wheelchair by type.

Despite the magnitude of the variance between VISNs, specific trends or patterns of provision of wheelchairs and scooters across VISNs could not be identified. For example, VISNs 1 and 10 provided a higher percentage of lightweight manual wheelchairs than standard manual wheelchairs and VISN 22 provided the highest percentage of ultralightweight wheelchairs. One might expect that VISNs 1,10 , and 22 would also provide a higher percentage of higher-end power wheelchairs. This, however, was not the case. VISNs 1 and 22 provided a relatively low percentage of power wheelchairs and a moderate percentage of scooters; VISN 10 provided rather high percentages of standard power wheelchairs and scooters. VISN 9 provided a relatively high proportion of custom power wheelchairs (6\%) and ultralightweight manual wheelchairs (4\%) but also provided a relatively higher proportion of standard manual wheelchairs (66\%). The six VISNs that provided relatively high proportions of standard power wheelchairs (5\%-7\%) did not necessarily provide a higher proportion of standard manual wheelchairs.

Based on the results of this study, our investigative team has hypothesized (for future studies) that factors affecting variation in the provision of wheelchairs and scooters occur at three levels: (1) data entry, (2) patient, and/or (3) VA system/VA facility. We earlier documented the variance in data entry. At the patient level, if age were driving variation across VISNs, one would expect age to vary across VISNs. This, however, was not the case. The mean ages of the veterans were stable across VISNs and ranged from 66.6 to 68.4 years. A previously published study that used this same cohort also found that age did not drive variation in WC/S provision to veterans [8]. Furthermore, this finding also holds true in the study we are currently conducting, in which we control for diagnosis and severity of disability. 
Although diagnosis has been identified as a potential confounding factor for type of device provided [8], in this study, the diagnoses of the cohort were stable across VISNs: ALS ranged from 1 to 2 percent, multiple sclerosis and Parkinson's disease from 3 to 4 percent, amputation from 4 to 5 percent, arthritis from 11 to 12 percent, and stroke from 15 to 17 percent. Thus, diagnosis does not appear to be driving the inconsistent WC/S provision across VISNs. SCI was the most variable diagnosis, ranging from 5 to 10 percent; however, the VISNs with higher percentages of veterans with SCI were not the VISNs that provided the highest percentages of ultralightweight manual wheelchairs, as might be expected. A limitation of having only diagnostic information, however, is that severity of disability remains unknown. A present study by our investigative team is examining the relationship between device provided and severity of disability.

Variance at the VA-facility level may also affect the provision of assistive devices, e.g., philosophical differences at the administrative and clinician levels and variation in the type of facility prescribing the device, including staffing and specialization. We have performed preliminary explorations of system-level variation, with more formal investigations planned. Future studies will investigate the extent to which VA staffing patterns affect variation in the type of WC/Ss provided.

Training in seating and mobility is a VA-system/VAfacility factor that can affect provision of mobility devices. A study is currently underway that compares VA and Medicare provision patterns. Medicare has recognized the importance of clinician and provider training in the wheelchair provision process, as reflected in the CMS announcement that after April 1, 2008, certain power wheelchairs and push-rim activated power-assist devices for manual wheelchairs cannot be provided until a Rehabilitation Engineering and Assistive Technology Society of North America (RESNA)-certified Assistive Technology Practitioner specializing in wheelchairs or a physician board-certified in Physical Medicine and Rehabilitation has performed an evaluation. Further, CMS has stipulated that the wheelchair must be provided by a RESNAcertified Assistive Technology Supplier who specializes in wheelchairs and is directly involved in the patient's wheelchair selection.

An additional system-level factor that potentially affects WC/S provision is implementation of VA purchasing contracts as a cost-saving measure. During
FY00-FY01, a contract for the purchase of standard manual wheelchairs was implemented, which may have led to a tendency to purchase the contracted standard wheelchairs. The VA has since expanded their contract options so that many wheelchair types can be purchased for the contract price.

\section{CONCLUSIONS}

This project is the first known national study to investigate VHA costs in providing WC/Ss and to compare prescription patterns across VISNs. During FY00 and FY01, the VHA provided more than 131,000 WC/Ss, at a cost of $\$ 109,010,198$. The most frequently provided type of wheeled device during this 2-year period was the standard manual wheelchair (53\%), followed by the lightweight rehabilitation manual wheelchair (17\%), and then the scooter (13\%). The types of WC/Ss provided across VISNs varied considerably and could not be explained by age or diagnosis.

While the data described here do not produce clear patterns of provision, they do support geographic variation and suggest that, while some facilities within VISNs may be overprescribing, others may be underprescribing. As the availability of and demand for wheeled mobility devices increase, two important issues must be addressed: (1) policy or clinical guidelines must be established to allocate the devices and (2) clinicians must be kept up-to-date in their training on this rapidly changing technology. The long-range goal of our research is the emergence of "best practices," so that appropriate use and equity in the provision of devices and quality of care will prevail.

\section{ACKNOWLEDGMENTS}

This material is the result of work supported by internal funds from the VA Rehabilitation Research and Development Center of Excellence on Wheelchairs and Related Technology, the VA Center of Excellence for Health Equity, the VA Predoctoral Associated Health Rehabilitation Research Fellowship Program, and the University of Pittsburgh Provost Predoctoral Fellowship Program.

The authors have declared that no competing interests exist. 


\section{REFERENCES}

1. Render ML, Taylor P, Plunkett J, Nugent GN. Methods to estimate and compare VA expenditures for assistive devices to Medicare payments. Med Care. 2003;41(6 Suppl):II70-79. [PMID: 12773829]

2. Downs F Jr. The National Prosthetic Patient Database: A national information resource for prosthetic prescriptions written in the VA. J Rehabil Res Dev. 2000;37(3):vii-xi. [PMID: 10917255]

3. Department of Veterans Affairs Information Resource Center (VIReC). Researcher's guide to VA data. Washington (DC): Department of Veterans Affairs; 2005.

4. Fitzgerald SG, Reker DM, Cowper DC, Hoenig H, Marshall C, Mann WC, Hubbard SL. Assessment of the National Prosthetics Database: Preliminary studies of validity. Pittsburgh (PA): VA Healthcare System; 2003.

5. Food and Drug Administration. Premarket notification [510(k)] [homepage on the Internet]. Rockville (MD): Center for Devices and Radiological Health, Food and Drug Administration; [updated 2006 Nov 1; cited 2007 Jul 11]. Available from: http://www.fda.gov/cdrh/devadvice/314.html

6. Center for Medicare and Medicaid Services. Procedures for coding and payment determinations for clinical laboratory tests and for durable medical equipment. Washington (DC): Center for Medicare and Medicaid Services; 2004.

7. Department of Veterans Affairs. Facility directory [homepage on the Internet]. Washington (DC): Department of Veterans Affairs; c2004 [updated 2006 Oct 31; cited 2007 Jul 11]. Available from: http://www1.va.gov/directory/ guide/home.asp?isFlash=1

8. Hubbard SL, Fitzgerald SG, Reker DM, Boninger ML, Cooper RA, Kazis LE. Demographic characteristics of veterans who received wheelchairs and scooters from Veterans Health Administration. J Rehabil Res Dev. 2006;43(7): 831-44. [PMID: 17436170]

9. Rubin RM, White-Means SI. Race, disability and assistive devices: Sociodemographics or discrimination. Int J Soc Econ. 2001;28(10-12):927-41.

10. Tomita MR, Mann WC, Fraas LF, Burns LL. Racial differences of frail elders in assistive technology. Assist Technol. 1997;9(2):140-51. [PMID: 10177451]

11. Hoenig H, Landerman LR, Shipp KM, George L. Activity restriction among wheelchair users. J Am Geriatr Soc. 2003;51(9):1244-51. [PMID: 12919236]
12. Fitzgerald SG, Cooper RA, Boninger ML, Rentschler AJ. Comparison of fatigue life for 3 types of manual wheelchairs. Arch Phys Med Rehabil. 2001;82(10):1484-88. [PMID: 11588758]

13. Cooper RA, Gonzalez J, Lawrence B, Renschler A, Boninger ML, VanSickle DP. Performance of selected lightweight wheelchairs on ANSI/RESNA tests. American National Standards Institute-Rehabilitation Engineering and Assistive Technology Society of North America. Arch Phys Med Rehabil. 1997;78(10):1138-44. [PMID: 9339166]

14. Rogers H, Berman S, Fails D, Jaser J. A comparison of functional mobility in standard vs ultralight wheelchairs as measured by performance on a community obstacle course. Disabil Rehabil. 2003;25(19):1083-88. [PMID: 12944147]

15. Kittel A, Di MA, Stewart H. Factors influencing the decision to abandon manual wheelchairs for three individuals with a spinal cord injury. Disabil Rehabil. 2002;24(1-3):106-14. [PMID: 11827144]

16. Hunt PC, Boninger ML, Cooper RA, Zafonte RD, Fitzgerald SG, Schmeler MR. Demographic and socioeconomic factors associated with disparity in wheelchair customizability among people with traumatic spinal cord injury. Arch Phys Med Rehabil. 2004;85(11):1859-64. [PMID: 15520982]

17. Boninger ML, Souza AL, Cooper RA, Fitzgerald SG, Koontz AM, Fay BT. Propulsion patterns and pushrim biomechanics in manual wheelchair propulsion. Arch Phys Med Rehabil. 2002;83(5):718-23. [PMID: 11994814]

18. Boninger ML, Baldwin M, Cooper RA, Koontz AM, Chan L. Manual wheelchair pushrim biomechanics and axle position. Arch Phys Med Rehabil. 2000;81(5):608-13. [PMID: 10807100]

19. Koontz AM, Cooper RA, Boninger ML. An autoregressive modeling approach to analyzing wheelchair propulsion forces. Med Eng Phys. 2001;23(4):285-91. [PMID: 11427366]

20. Van der Woude LH, Veeger HE, Dallmeijer AJ, Janssen TW, Rozendaal LA. Biomechanics and physiology in active manual wheelchair propulsion. Med Eng Phys. 2001;23(10): 713-33. [PMID: 11801413]

21. Department of Veterans Affairs. VHA Handbook. Washington (DC): Department of Veterans Affairs; 2002.

Submitted for publication October 24, 2006. Accepted in revised form April 27, 2007. 